\title{
Optimal Placement and Channel Assignment of Relay Stations in Heterogeneous Wireless Mesh Networks by Modified Bender's Decomposition
}

\author{
Aaron So and Ben Liang \\ Department of Electrical and Computer Engineering \\ University of Toronto \\ 10 King's College Road \\ Toronto, Ontario, M5S 3G4, Canada
}

\begin{abstract}
Fixed Broadband Wireless Access (FBWA) technology is designed to serve as a wireless DSL replacement to provide broadband Internet access in underserved areas where no other access technology exists. Due to the enormousness of the target service area, relay equipment play an important role in such networks, and the installation and maintenance cost of the network is directly proportional to the cost of the relay equipment. To minimize the network operational cost, an optimization framework which computes the minimum number of relay stations and their corresponding placements and channel assignments in the network is desired. Because the problem is NP-hard, we propose an efficient optimization algorithm based on a modified version of Bender's decomposition to iteratively compute converging bounds to the problem solution. Our numerical results show that by using a few relay stations in a rural community, broadband Internet access can be established in a cost effective manner.
\end{abstract}

Key words: Fixed broadband wireless access, relay stations, placement and channel assignment, Bender's decomposition, mathematical programming/optimization.

Email address: $\{$ aaronso, liang\}@comm. utoronto.ca (Aaron So and Ben Liang).

1 A preliminary version of this work was presented at the 2007 IFIP Networking Conference [1]. This work was supported in part by Bell Canada through its Bell University Laboratories R\&D program. 


\section{Introduction}

Broadband wireless has long held the promise of delivering a wide range of data and information services to business and residential customers quickly and costeffectively. With the publication of a comprehensive industry standard, namely IEEE 802.16, representing a distillation of the most advanced technology and an industry consensus permitting equipment interoperability, broadband wireless has gained maturity and is ready to unleash its full potential. In many instances, wireless broadband is the preferred access technology, offering the best cost/performance ratio, time to market, and service velocity. However, at this point, broadband wireless is still a divergent, even disruptive, technology, and wireline solutions such as fiber optics, hybrid fiber coax, and digital subscriber line (DSL) constitute the mainstream. For this reason, niche market has to be identified and innovative yet cost effective strategies have to be invented to make broadband wireless more attractive to broadband Internet service providers.

Since the propagation characteristics of radio waves are so different in the lowerand upper-microwave regions, separate specifications are required for any broadband wireless access technologies using these spectrums. For example, the IEEE 802.16 standard has two separate physical layer specifications. The WirelessMANOFDM specifications utilize the $2-11 \mathrm{GHz}$ spectrum, while the WirelessMAN-SC specifications utilize the 10-66 GHz spectrum. Lower frequency signals can penetrate walls and deflect from obstacles, while higher frequency transmissions must meet strict line-of-sight (LOS) requirements. However, this strict LOS requirement facilitates the efficient usage of directional antenna array technologies and virtually eliminates the effect of interference. In addition, the advantage of using high frequency bands is an abundance of bandwidth. These intrinsic properties of IEEE 802.16 technology or any broadband access technologies which employ both the upper- and lower-microwave spectrum make them ideal for a heterogeneous architecture.

An example of such architecture is illustrated in Fig. 1. In this network, we assume a base station wired to the ISP core network, and this base station is assigned to serve subscribers in a particular area. Because of the size of the coverage area, the base station usually cannot serve every subscriber by a single-hop communication. As a result, several relay stations (RSs) are installed in the network, for example, on the subscribers' rooftops, to relay traffic from and to the base station. If LOS communications can be established among some RSs and the base station, the bandwidth abundant upper spectrum is used to form a backbone network. The lower spectrum, which can be divided into a fixed number of channels, on the other hand, is used by the base station and RSs to communicate directly with the subscribers and form the corresponding local network. Since the RSs have to be equipped with technologies which handle communications from both the upper- and lower-microwave spectrum, they are usually expensive. The focus of this work is to minimize the number 


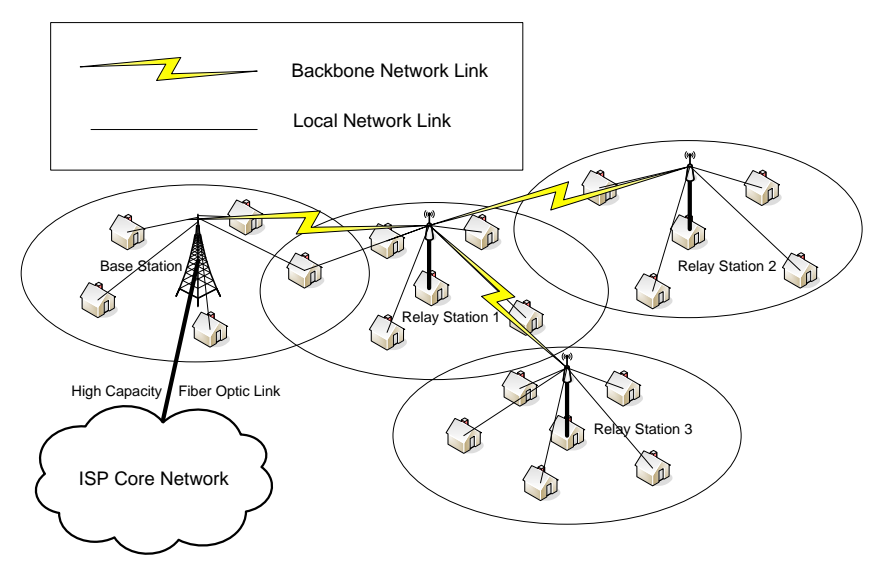

Fig. 1. Heterogeneous Wireless Mesh Network.

of RSs used in the mesh network while maintaining the pre-specified uplink and downlink demands of the subscribers.

To the best of our knowledge, this work is among the first solutions to address the problem of joint relay equipment placement and channel assignment in a heterogeneous wireless mesh network. Specifically, the contributions of this paper are as follows. First, we describe a heterogeneous wireless mesh network architecture with relay infrastructure which utilizes the intrinsic properties of broadband wireless access technologies employing the upper- and lower-microwave spectrum. Second, we develop an analytical framework which calculates the cost and feasibility of a network with a particular relay station placement and channel assignment. Third, we propose an optimization framework based on Bender's decomposition to calculate the minimum deployment and maintenance cost of a given heterogeneous wireless mesh network. Finally, we propose a combination of heuristic and an objective-cut method to reduce the run time of the Bender's decomposition method.

The rest of this paper is organized as follows. In Section 2, we review the related work in multihop wireless networks. In Section 3, we describe the network infrastructure and equipment capabilities. In Section 4, we define our relay station placement and channel assignment problem mathematically, and describe our optimization solution. In Section 5, we present physical layer rate models for the IEEE 802.16 specifications under a large scale propagation model. In Section 6, we discuss the convergence time and performance of the proposed optimization algorithm. Finally, concluding remarks are given in Section 7.

\section{Related Works}

Motivated by recent advances in wireless ad hoc networking [2][3], there has been much research into using multihop communication in the context of wireless net- 
works. In particular, wireless multihop mesh networks have attracted much attention in recent years. Departing from the traditional single hop architecture, wireless multihop networking is now considered as the next evolutionary step for wireless data networks. In [4], Dong et al. showed that the objective of improving throughput without sacrificing fairness can be much better met in multihop WLANs than in single-hop WLANs. In [5], Draves et al. proposed to use several novel decision metrics to determine routes in a multi-radio, multihop network. Experiments were performed in a wireless testbed, and promising results were reported. These results show the practicality of the wireless mesh network architecture.

To bring wireless mesh networks closer to reality, the fundamental properties of a wireless mesh network must be well understood. In [6], Draves et al. conducted a detailed empirical evaluation of several link-quality metrics on route computation performance. Kodialam and Nandagopal discussed the fundamental quantity of achievable bit rate in multihop wireless mesh networks with orthogonal channels in [7]. Tight necessary and sufficient conditions for rate achievability were developed. In [8], an analytical model was developed by Waharte and Boutaba to understand the end-to-end delay in a tree-based mesh network. Furthermore, in [9], Robinson et al. presented a methodical approach that offers a way to calibrate wireless mesh network testbeds before experimental deployment and evaluation.

The issue of interference management in wireless mesh networks has been discussed in several contexts. In [10], Jain et al. considered the fundamental question of how much throughput a given wireless mesh network can achieve under different interference conditions. To address operational issues, an interference-aware channel assignment algorithm for multi-radio wireless mesh networks was proposed in [11] by Ramachandran et al. Moreover, in [12], an interference-aware cross-layer design was proposed by Wei et al. to increase the throughput of an IEEE 802.16 wireless mesh network. To take advantage of advance technologies, studies of using directional antennas to control interference in a wireless mesh network were performed by Kumar et al. in [13]. The results of these works provide insight into the impact of interference in wireless mesh networks.

The problem of wireless network equipment placement has also been addressed in several works. In the context of sensor networks, the optimal number and placement of sensors which maximizing the network lifetime are discussed by Chen et al. in [14]. In [15], So and Liang proposed an Lagrangian approach to computes the optimal placement of a fixed number of relay nodes, which relay traffic in a twohop fashion, to improve throughput in a wireless local area network. In the context of community mesh networks, innovative integration techniques were developed by Begerano in [16] to minimize the number of access points in a mesh network to reduce wiring cost, while maintaining QoS constraints from all nodes. A similar problem was discuss by Chandra et al. in [17] under different link and network models. From the results of these works, we learn that the placement of wireless network equipments can have significant impact on network performance. To the 
best of our knowledge, there is no existing work that addresses the problem of joint relay equipment placement and channel assignment in community wireless mesh networks, which is what we investigate in this work.

\section{Network Infrastructure and Equipment Capabilities}

To establish a network in a rural area, an operator needs to establish a site for the initial base station and the central office, which should have high capacity backhaul connection with the ISP core network. One cost effective backhaul solution is to lease dark fiber from a public utility or public transit system. Gas and electrical utilities and railroad companies own extensive amounts of optical fiber that they use for monitoring purposes and internal communications. Such private fiber networks are generally grossly underutilized, and the owners are often amenable to leasing capacity. However, since such private network is already established, the network point of access is already fixed. Thus, the ISP does not have the freedom to choose the location of the central office and initial base station. In this work, we consider the case where the location of the base station and central office is given.

After the base station and central office are established, the operator needs to obtain roof rights and estimate the demands of potential subscribers. The operator can accomplish them by negotiating with property owners and surveying Internet demands of potential subscribers in the target service area. In this work, we consider the case where the set of exploitable roofs and the demands of potential subscribers are given as the input of the problem.

Given the input to the system, our goal is to place the minimum number of relay stations in the network such that the demands of the subscribers can be met. Since the subscriber locations are fixed and the high frequency spectrum is used, by using adaptive array antenna technologies and the three-dimensional space intelligently, we can effectively control interference in both the backbone and local networks. Adaptive array antenna technologies have the ability to focus a beam very tightly toward a receiver. In some systems [18][19], the adaptive array antenna can detect interfering source and use vector-cancelation strategies to create a null beam to cancel the interfering source. In one experimental system developed by Lucent [20], the array antenna system could simultaneously receive several signals over the same channel by reconstructing them based on their time of arrival and multipath signature. By combining this advanced technology with the upper microwave spectrum, there is virtually no interference in the backbone network. However, an adaptive array antenna system requires multiple antenna elements and heavy duty processors. To date, the cost of such equipment is high. Thus, in this work, we only assume the backbone network uses such equipment.

For the local network, the strict LOS requirement cannot be assumed. Thus, the 


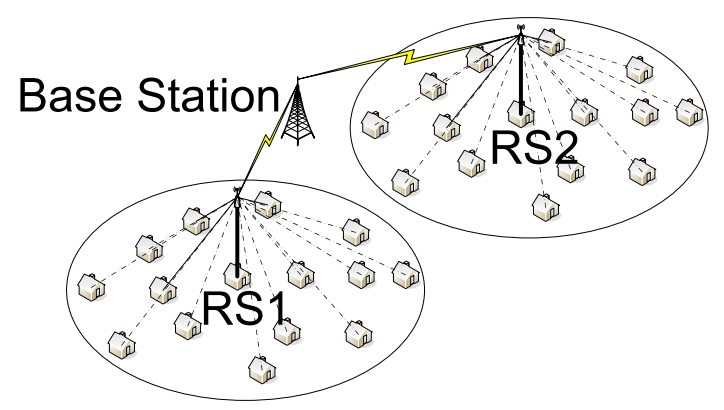

Fig. 2. Tilted polarized directional antenna systems.

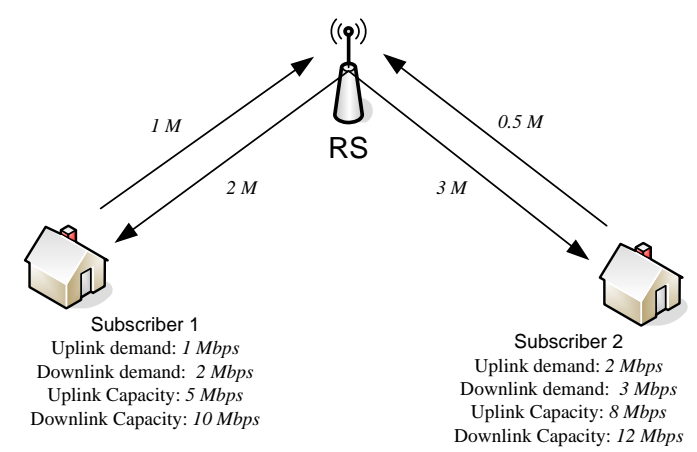

Fig. 3. Example of local network capacity.

lower-microwave spectrum is used. Since the above adaptive antenna array systems are expensive and they were designed mainly for the upper-microwave spectrum, they are not suitable for the local networks. In this work, we assume a more affordable and common approach [20]: polarized directional antennas. In this case, the antenna system is polarized so that it only disposes magnetic fields horizontally. When the antenna is tilted downward (or upward), beyond a certain distance, the radiation will simply be absorbed into the ground (or outer space). As shown in Fig. 2, two relay stations can use the same channel for the local network and do not interfere with each other as long as they are placed far apart from each other. However, since subscribers who use the same RS are located in the same multipath environment, they have to share the channel in a time-multiplexed fashion while not exceeding the capacity of the RS. Fig. 3 illustrates a simple example. Each subscriber has its prespecified uplink and downlink demands, and the local link capacities between a subscriber and the RS are also given. For example, subscriber 1 has to transmit $1 M$ bits every second to the RS via a $5 M b p s$ link. The time to transport these $1 M$ bits is $1 / 5=0.2$ second. Similarly, the time to transport the downlink data of subscriber 1, the uplink data of subscriber 2, and the downlink data of subscriber 2 are $0.2,0.25$, and 0.25 second respectively. Thus, the total time of transporting all these data is $0.2+0.2+0.25+0.25=0.9$ second, which is less than 1 second. (i.e. it does not exceed the capacity of the RS.) In the next section, we define the problem mathematically. 


\section{System Model and Optimization Framework}

Suppose there are $N$ subscribers and one base station in the system, and they are represented by the set $V=\{0,1, \ldots, N\}$, where the base station is represented by the index 0 . Let $V_{R} \subseteq V$ be the set of nodes where the installation of relay stations are feasible. ${ }^{2}$ We can use the set $V_{R}$ to form a directed-complete graph representing the backbone network. The link weight from node $i$ to node $j$, denoted $C_{i j}^{B}$, represents the capacity in terms of bit per second from node $i$ to node $j$ using backbone technology. The capacity between two nodes is zero if line-of-sight communication cannot be established or the distance between them is too high such that communication is impossible.

Similar to the backbone network, we can use the set $V$ to form a directed-complete graph representing the local network. Within the local technology, the capacity from node $i$ to node $j$ is denoted by $C_{i j}^{L}$. Since a link which handles local traffic has to be associated with the base station or a relay station, $C_{i j}^{L}=0$ if both $i$ and $j$ are not in the set $V_{R}$. Moreover, the capacity between two nodes is also zero if the distance between them is too high.

As mentioned above, two relay stations using the same channel would interfere each other's local network operations, if they are placed in each other's interfering zones. Let $N(i)$ be the set of nodes that interfere the operation of node $i$, where $i \in$ $V_{R}$. Moreover, when there are $N_{C}$ available local channels, let $\Lambda=\left\{1,2, \ldots, N_{C}\right\}$ be the local channel set. Furthermore, for each subscriber $i$, there is a pre-specified uplink demand, $u_{i}$, and downlink demand, $d_{i}$. Given the above as the input to our problem, we define the following decision variables.

$$
\begin{aligned}
X_{i}^{\lambda} & =\left\{\begin{array}{l}
1 \text { if an RS which uses channel } \lambda \text { is installed at node } i . \\
0 \text { otherwise }
\end{array} \quad i \in V_{R}, \lambda \in \Lambda\right.
\end{aligned}
$$

Note that all input and decision variables are non-negative. Moreover, we define $X_{0}^{1}=1$ since the base station is always present, and without loss of generality, we can also let the base station to use channel 1 for its local network operation. Next, we formulate our problem as a non-linear mixed integer program.

2 Whether a subscriber site is in $V_{R}$ or not depends on the willingness of the subscriber and other physical conditions. Furthermore, the base station, which has index 0 , is included in the set $V_{R}$. 


\subsection{Optimization Formulation}

Our goal is to find the minimum number of RSs in the system which satisfies all the subscribers' demand and network constraints. The optimization formulation is as follows:

$$
\begin{aligned}
& \min _{\mathbf{X}}: \sum_{i \in V_{R}} \sum_{\lambda \in \Lambda} X_{i}^{\lambda} \\
& \text { s.t. } \sum_{i \in V_{R} \backslash\{0\}} f_{i 0}^{u}+\sum_{i \in V \backslash\{0\}} h_{i 0}^{u}=\sum_{i \in V \backslash\{0\}} u_{i} \\
& \sum_{i \in V_{R} \backslash\{0\}} f_{0 i}^{d}+\sum_{i \in V \backslash\{0\}} h_{0 i}^{d}=\sum_{i \in V \backslash\{0\}} d_{i} \\
& \sum_{j \in V_{R}, i \neq j} f_{j i}^{u}+\sum_{j \in V \backslash\{0\}} h_{j i}^{u}=\sum_{j \in V_{R}, i \neq j} f_{i j}^{u} \quad \forall i \in V_{R} \backslash\{0\} \\
& \sum_{j \in V_{R}, i \neq j} f_{j i}^{d}-\sum_{j \in V \backslash\{0\}} h_{i j}^{d}=\sum_{j \in V_{R}, i \neq j} f_{i j}^{d} \quad \forall i \in V_{R} \backslash\{0\} \\
& \sum_{j \in V_{R}} h_{i j}^{u} \geq u_{i} \quad \forall i \in V \backslash\{0\} \\
& \sum_{j \in V_{R}} h_{j i}^{d} \geq d_{i} \quad \forall i \in V \backslash\{0\} \\
& \sum_{\lambda \in \Lambda} X_{i}^{\lambda} \leq 1 \quad \forall i \in V_{R} \\
& \sum_{j \in V, j \neq i} \frac{h_{i j}^{d}+h_{i j}^{u}}{C_{i j}^{L}}+\frac{h_{j i}^{d}+h_{j i}^{u}}{C_{j i}^{L}} \leq\left(1-\sum_{\lambda \in \Lambda} X_{i}^{\lambda}\right) k+1 \quad \forall i \in V_{R} \\
& \sum_{j \in V} h_{i j}^{d}+h_{j i}^{u} \leq k \sum_{\lambda \in \Lambda} X_{i}^{\lambda} \quad \forall i \in V_{R} \\
& f_{i j}^{u}+f_{i j}^{d} \leq C_{i j}^{B} \sum_{\lambda \in \Lambda} X_{i}^{\lambda} \sum_{\lambda \in \Lambda} X_{j}^{\lambda} \quad \forall i \in V_{R}, j \in V_{R}, i \neq j \\
& X_{i}^{\lambda}+\sum_{j \in N(i)} X_{j}^{\lambda} \leq 1 \quad \forall i \in V_{R}, \lambda \in \Lambda
\end{aligned}
$$

The objective (1) minimizes the number of RSs to be installed in the network. Constraints (2) and (3) verify that the amount of traffic entering and exiting the base station equals the total uplink and downlink demands respectively. Constraints (4) and (5) verify that the amount of traffic entering each RS matches the amount of traffic exiting each RS (the conservation of flow at each RS). Constraints (6) and (7) verify that the uplink and downlink demands are met respectively. Constraint (8) verifies that at most one channel can be assigned to an RS. Constraints (9) and (10) work together with an arbitrary large number $k$. If an RS is placed at node $i$, then $\sum_{\lambda \in \Lambda} X_{i}^{\lambda}=1$, and the right hand side of constraint (9) is 1. Since local uplink and downlink traffic share the channel in a time-multiplexed fashion, constraint (9) verifies that the local traffic enters and exits through the $i^{\text {th }} \mathrm{RS}$ does not exceed its 
capacity. If no RS is installed at node $i$, then $X_{i}^{\lambda}=0 \forall \lambda \in \Lambda$ and the right hand side of constraint (9) is $k+1$. Thus, constraint (9) does not impose any restriction on the traffic exiting and entering node $i$. However, the right hand side of constraint (10) is 0 . This ensures that no local uplink traffic enters node $i$ and no local downlink traffic exits node $i$. Constraints (11) ensures that a positive backbone traffic between node $i$ and $j$ exists only if an RS is placed at node $i$ and an RS is placed at node $j .{ }^{3}$ Finally, constraint (12) ensures that no two RSs which use the same channel are placed in each other's interfering zone. Our goal is to find the minimum number of RSs in the system which satisfies all the demand and interference constraints. The optimization formulation is as follows:

\subsection{Problem Reformulation and Bender's Decomposition}

The above problem is similar to two problems in location theory [21][22]: the hub location problem and the maximum coverage problem. An RS functions as a hub and a service node. Thus, the resulting problem is a hybrid of these two well-known location problems. However, to the best of our knowledge, there is no existing solution to this hybrid problem. Moreover, due to the discrete and non-linear nature of the problem, explicit enumeration, a.k.a. exhaustive search, is the only available solution. By using this method, an exponential number of linear program has to be solved, which makes this approach intractable. However, given that we have a large number of continuous variables and a relatively small number of integer variables, with a simple but essential reformulation, this problem can be solved by Bender's Decomposition. Bender's decomposition breaks down the problem to a sequence of small 0-1 integer problems [23] which can be solved efficiently by commercial optimization softwares such as CPLEX or well-known techniques such as branchand-bound. In the following, we first reformulate the above analytical framework so that it can be decomposed by Bender's method. Then we describe the algorithm that we used to solve the RS placement and channel assignment problem.

First, to apply Bender's decomposition to a mixed integer problem, all constraints have to be linear. Since constraint (11) is not linear, we replace Constraint (11) by the following two constraints.

$$
\begin{aligned}
& f_{i j}^{u}+f_{i j}^{d} \leq C_{i j}^{B} \sum_{\lambda \in \Lambda} X_{i}^{\lambda} \\
& f_{i j}^{u}+f_{i j}^{d} \leq C_{i j}^{B} \sum_{\lambda \in \Lambda} X_{j}^{\lambda}
\end{aligned}
$$

Second, Bender's method requires a mixed integer program to satisfied a certain feasibility assumptions and to be organized into the following form

3 Note that constraint (11) is a non-linear constraint. 


$$
\begin{aligned}
& \min _{x} D \\
& \text { s.t. } D \geq c x+(b-A x)^{t} u_{i} \quad \forall i \in[1, p],
\end{aligned}
$$

where $x$ is the vector representing the discrete decision variables, $c$ is the vector associate with $x$ in the objective, $A$ is the matrix associate with $x$ in the constraint set, $b$ is the constant vector for the constraint set, and $u_{i}$ is a constant vector for the $i^{\text {th }}$ constraint.

What we have from (1) - (12) can be written as ${ }^{4}$

$$
\begin{aligned}
& \min _{x, y} c_{1} y+c_{2} x \\
& \text { s.t. } A_{1} y+A_{2} x \geq b,
\end{aligned}
$$

where $x$ is a vector represents the location-channel variables $X_{i}^{\lambda}, y$ is a vector represents the set of continuous variables $f_{i j}^{u}, f_{i j}^{d}, h_{i j}^{u}, h_{i j}^{d}, c_{1}=\mathbf{0}^{t}, c_{2}=\mathbf{1}^{t}$, and $(\cdot)^{t}$ denotes vector transposition.

For a fixed value of the location-channel variables $x=\widehat{x}$, problem (17) reduces to the following constraint-violation problem:

$$
\begin{aligned}
& \min _{y} T(y \mid \widehat{x}) \triangleq c_{1} y \\
& \text { s.t. } A_{1} y \geq b-A_{2} \widehat{x}
\end{aligned}
$$

Obviously, given a particular RS placement and channel assignment, $\widehat{x}$, the resulting problem, (19)(20), may or may not be feasible. This violates the assumptions of Bender'e method. To make all location-channel variables feasible, let us introduce one positive continuous variable, $v$, and a very large infeasibility constant, $P$. We can then modify (19)(20) by changing $A_{1}$ to $A_{1^{\prime}}=\left[A_{1} \mid \mathbf{1}\right], c_{1}$ to $c_{1^{\prime}}=\left[\mathbf{0}^{t} \mid P\right]$, and $y$ to $y^{\prime}=y \bigcup v$. Then, the modified constraint-violation problem is the following:

$$
\begin{aligned}
& \min _{y^{\prime}} T\left(y^{\prime} \mid \widehat{x}\right)=c_{1^{\prime}} y^{\prime}=P v \\
& \text { s.t. } A_{1^{\prime}} y^{\prime} \geq b-A_{2} \widehat{x}
\end{aligned}
$$

For any $\widehat{x}$ which makes (19)(20) infeasible, problem (21) (22) is still feasible, but it will suffer a very large infeasible penalty $P v$.

Now, let us now consider the dual of the modified constraint-violation problem (21)(22). Let $u$ be the set of dual variables. The dual of the modified constraintviolation problem may now be formulated as follows:

$\overline{4 a=b}$ is equivalent to $a \geq b$ and $b \geq a$. 


$$
\begin{array}{rl}
\max _{u} & D(u \mid \widehat{x}) \triangleq\left(b-A_{2} \widehat{x}\right)^{t} u \\
\text { s.t. } & A_{1^{\prime}}^{t} u \leq c_{1^{\prime}}^{t} \\
& u \geq 0
\end{array}
$$

Denote the optimal solutions to the linear programs (21) and (23) by $y^{\prime *}$ and $u^{*}$ respectively. Then, by duality theory,

$$
c_{1^{\prime}} y^{\prime *}=\left(b-A_{2} \widehat{x}\right)^{t} u^{*}
$$

We now consider all the extreme points of the dual problem (23). Note that the extreme points are defined by the feasible region described by (24) and (25) which are independent of the location-channel variables $x$. Thus, the extreme points can be generated without any knowledge of the RS locations and channel assignments. Let us denote the $i^{\text {th }}$ extreme point by $u_{i}$ and total number of extreme points by $p$. We know from the theory of linear programming that at least one optimal solution to any linear problem occurs at an extreme point of the feasible region. Thus, problem (21)(22) can be reformulate as the following pure 0-1 problem:

$$
\begin{aligned}
& \min _{x} c_{2} x+D \\
& \text { s.t. } D \geq\left(b-A_{2} x\right)^{t} u_{i} \quad \forall i \in[1, p],
\end{aligned}
$$

or equivalently,

$$
\begin{aligned}
& \min _{x} D^{\prime} \\
& \text { s.t. } D^{\prime} \geq c_{2} x+\left(b-A_{2} x\right)^{t} u_{i} \quad \forall i \in[1, p] .
\end{aligned}
$$

which has the desired form as in (15)(16). Now, the problem is reformulated to a form that can be solved by Bender's method. The difficulty with problem (29) is that the number of extreme points of the dual problem is potentially very large. Thus, we do not want to enumerate all of the constraints in (30) explicitly. Also, at the optimal solution to (29), only a small subset of the constraints (30) are likely to be tight. Thus, even if we could enumerate all of them, many of them would prove to be unnecessary. On the other hand, if we solve (29) with only a subset of the constraints in (30), we will obtain a valid lower bound on the optimal value of the original objective function. Furthermore, if all of the constraints that are tight in the optimal solution to (29) happen to be in the subset of constraints that we include, then the value of the objective function (29) will exactly equal the optimal value. In the following subsection, we present the Bender's method which generates the desired subset of extreme points iteratively. 


\subsection{Bender's Method}

To generate the desired subset of extreme points, Bender's method adds constraints to the constraint set (30) one by one [23]. When a new constraint is added, the optimal solution of (29) either returns a better (larger) lower bound value or the optimal solution to the original problem (17) if a feasible RS placement and channel assignment exists. ${ }^{5}$ Bender's method is an iterative algorithm. At each iteration, the algorithm solve one linear program, which can be solved efficiently by simplex method, and one small pure 0-1 integer program, which can be solve efficiently by well-known techniques like branch-and-bound. In the following we describe the initialization and the iterative steps of the algorithm.

\subsubsection{Step 0: Initialization}

To initialize problem (29)(30), we start with an empty constraint set (30) and an arbitrary location-channel variable $\widehat{x}_{0}$. The goal of this step is to find the first constraint to add to the constraint set (30) and to find an incumbent upper bound. To find the first constraint, we use the arbitrary location-channel variable $\widehat{x}_{0}$ to solve the dual of the modified constraint-violation problem (23) and obtain the extreme point $u_{0}$. In turn, we obtain the first constraint to add to (30): $D^{\prime} \geq$ $c_{2} x+\left(b-A_{2} x\right)^{t} u_{0}$. Moreover, we obtained the incumbent upper bound, $B_{U}(1)=$ $c_{2} \widehat{x_{0}}+\left(b-A_{2} \widehat{x_{0}}\right)^{t} u_{0}$ and incumbent solution $x^{*}=\widehat{x}_{0}$. In addition, we set the iteration number $i=1$.

\subsubsection{Step 1: Solve a small 0-1 integer program and obtain a lower bound}

As mentioned in the previous subsection, when constraint set (30) contains a subset of the desired constraints, the result of problem (29) gives a valid lower bound. As the constraint set grows, the lower bound converges to the optimal solution. In this step, we need to solve a small 0-1 integer program (29) and obtain the resulting lower bound value, $B_{L}(i)$, and the location-channel variable $\widehat{x}_{i}$ of this iteration.

\subsubsection{Step 2: Optimality Test}

If $B_{L}(i)=B_{U}(i)$, we have reached the optimal solution, and $x^{*}$ represents the optimal RS placement and channel assignment. In this case, we can stop; otherwise, we proceed to step 3 .

5 If no feasible solution exists, the algorithm will return a very large number. 


\subsubsection{Step 3: Solve a linear program and generate a new constraint}

The algorithm enters this step only if the lower bound is smaller than the upper bound. To improve the lower bound value, a new constraint, which is a half space, is needed to cut away $\widehat{x}_{i}$ from the solution space defined by (30). To achieve this, we use $\widehat{x}_{i}$ to solve the dual of the modified constraint-violation problem (23) and obtain the new extreme point $u_{i}$. In turn, we obtain a new constraint to add to (30), $D^{\prime} \geq c_{2} x+\left(b-A_{2} x\right)^{t} u_{i}$, and an upper bound value, $B_{U}=c_{2} \widehat{x_{i}}+\left(b-A_{2} \widehat{x_{i}}\right)^{t} u_{i}$. At this point we increment $i$ to $i+1$. If $B_{U}$ is smaller than the incumbent upper bound $B_{U}(i-1)$, we set $B_{U}(i)=B_{U}$ and $x^{*}=x_{i}$. Otherwise, we set $B_{U}(i)=B_{U}(i-1)$. Finally, we go back to step 1 .

\subsection{Modified Bender's Method}

In the previous subsection, we demonstrated a tractable solution based on Bender's method to solve the relay station placement and channel assignment problem. Even though this approach makes the problem manageable, it could be time consuming. In this subsection, we exploit the intrinsic properties of this problem and develop techniques which may potentially speed up the run time of the original Bender's method.

As described in the previous subsection, the key of Bender's method is to generate constraints, reduce the solution space (30), and generate better (larger) lower bound iteratively. If we can generate constraints more rapidly or further reduce the solution space after getting some information from the results of each iteration, we can potentially reduce the run time of the algorithm.

First, we can take advantage of the integer nature of the objective value in the original problem to further reduce the solution space of (30). To illustrate our technique, let us consider a simple example. If the incumbent upper bound is 12.8 and the lower bound is 8.2, we know that the optimal number of RSs must be 9, 10, 11, or 12. To express this idea mathematically, we can add the following constraints to (30).

$$
\left\lceil B_{L}(i)\right\rceil \leq \sum_{i \in V_{R}} \sum_{\lambda \in \Lambda} X_{i}^{\lambda} \leq\left\lfloor B_{U}(i)\right\rfloor .
$$

By adding this constraint, we may further reduce the solution space of the problem after each iteration, and accelerate the convergence of the solution.

Second, it is obvious that the most time consuming step of the Bender's method is Step 1 in which a small 0-1 integer program has to be solved. The purpose of finding the solution of (29) at each iteration is to use the resulting solution $\widehat{x}_{i}$ to find an appropriate extreme point, $u_{i}$ and add a new constraint to constraint set (30) in Step 3. Instead of performing an optimization at each iteration, we propose to

use heuristic to find a decent suboptimal solution and its corresponding extreme 
point, at each iteration, and only perform a minimization when the objective value generated by the heuristic is larger than or equal to the upper bound. In this work, we developed a slightly modified version of a simple descent method which incorporates the intrinsic structure of this problem. Before we describe the developed heuristic in the next subsection, let us describe the details of the modified Bender's method in Fig. 4.

\subsubsection{Step 0: Initialization}

For our proposed modified version of Bender's method, the initialization procedure is almost identical to that of the original method. The only difference is that an additional data structure $B_{E L}$ is initialized to record the estimated lower bound generated by the heuristic.

\subsubsection{Step 1: Solve a small 0-1 integer program and obtain an estimated lower bound}

A significant amount of modifications are added to this step. Instead of performing an optimization in each iteration, we try to generate a large amount of descent extreme points rapidly by heuristic, and only perform optimization if the result generated by the heuristic is invalid (larger than the upper bound) or may be optimal (equal to the upper bound). At each iteration, a heuristic is used to find a suboptimal solution for (29). The result generated by the heuristic in iteration $i$ is stored in $B_{E L}(i)$. If the suboptimal solution is smaller than the upper bound, we know that it cannot be the optimal solution. Thus, we can skip the optimality test in step 2 and proceed directly to step 3 . If the suboptimal solution is larger than the upper bound, we know the solution is invalid. Moreover, if the suboptimal solution is equal to the upper bound, it may be the optimal solution. In both cases, we perform an optimization and obtain the real lower bound for the current iteration. If an optimization is performed, we proceed to Step 2, the optimality test.

\subsubsection{Step 2: Optimality Test}

Identical to the original method.

\subsubsection{Step 3: Solve a linear program and generate a new constraint}

Identical to the original method. 

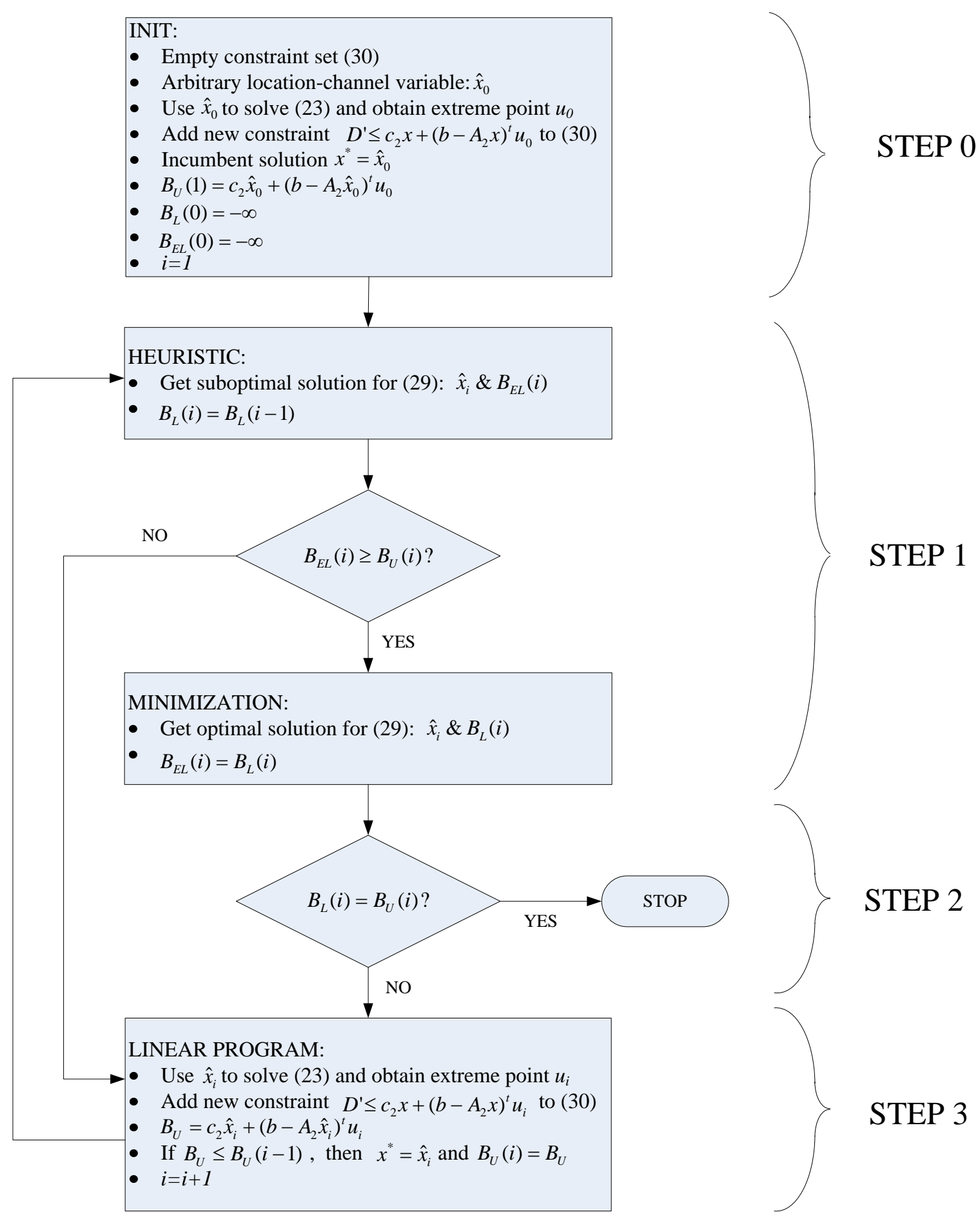

Fig. 4. Flowchart of the modified Bender's decomposition approach for solving the RS placement and channel assignment problem.

\subsection{Heuristic Used in the Modified Bender's Method}

It has been well recognized that heuristics can usually solve integer problems in a very short amount of time and produce reasonable solutions. In this work, we 
proposed to use a simple descent method which incorporates the intrinsic properties of the RS placement and channel assignment problem to solve (29) in each iteration. Like all descent methods, we have to define the neighborhood of a solution. For a particular integer solution for this problem, $\widehat{x}$, we can define its neighborhood be another solution with one of the following 4 operations performed:

(1) RS ADDITION: For a particular solution, if the set of variables, $X_{i}^{1}, \ldots, X_{i}^{N_{C}}$ are all zeros, that means no $\mathrm{RS}$ is installed at node $i$. If we ADD an RS, we set one of these bit to 1 .

(2) RS REMOVAL: Like RS Addition, for a particular solution, if one of the variables in the set $X_{i}^{1}, \ldots, X_{i}^{N_{C}}$ is one, that means an RS is installed at node $i$. If we REMOVE an RS, we set that bit to 0 .

(3) RS CHANNEL CHANGE: If one of the variables in the set $X_{i}^{1}, \ldots, X_{i}^{N_{C}}$ is one, that means an RS is installed at node $i$ and it is using a particular channel. If we clear this bit and set another bit to 1, we perform a CHANNEL CHANGE.

(4) RS MOVE: An RS MOVE is just an RS REMOVAL follow by an RS ADDITION.

For a particular integer solution, we can generate its entire neighborhood by using the above four operations. In addition, the neighborhood of a solution has to satisfy the objective cut constraint (31). In Fig. 5, let us describe the descent method in details.

Let us define the objective value of (29) with the integer solution $\widehat{x}$ be $F(\widehat{x})$, the neighborhood of a solution $\widehat{x}$ be $S_{\widehat{x}}(\cdot)$, and the number of neighbor solutions of $\widehat{x}$ be $N_{\widehat{x}}$. We start with an arbitrary solution $\widehat{x}$, and store the best objective value obtained so far in $F^{*}$. We evaluate the neighborhood solutions one by one. If we find a solution which results in a better objective value, we set $\widehat{x}$ to this new neighborhood value, and repeat the process again. The process stop only if the number of iteration has reached the maximum allowed value, max_iteration, or we reach a solution where no neighborhood solution results in a better objective value.

\subsection{Validity of Modified Bender's Method}

The main idea of the Bender's Decomposition approach is to solve (29) with a subset of constraint in (30). If the desired subset of constraints are included in (30), the solution of (29) is equaled to that of the original problem. Thus, finding the desired subset of constraints is the key of the iterative algorithm.

Before we discuss the validity of the Modified Bender's Method, let us briefly discuss the validity of the original Bender's Method described in Subsection 4.3. By using the original Bender's Method, after each iteration, say $i$, we obtain a new integer solution, $\widehat{x_{i}}$, a lower bound, $B_{L}(i)$, a upper bound $B_{U}(i+1)$, and a new 


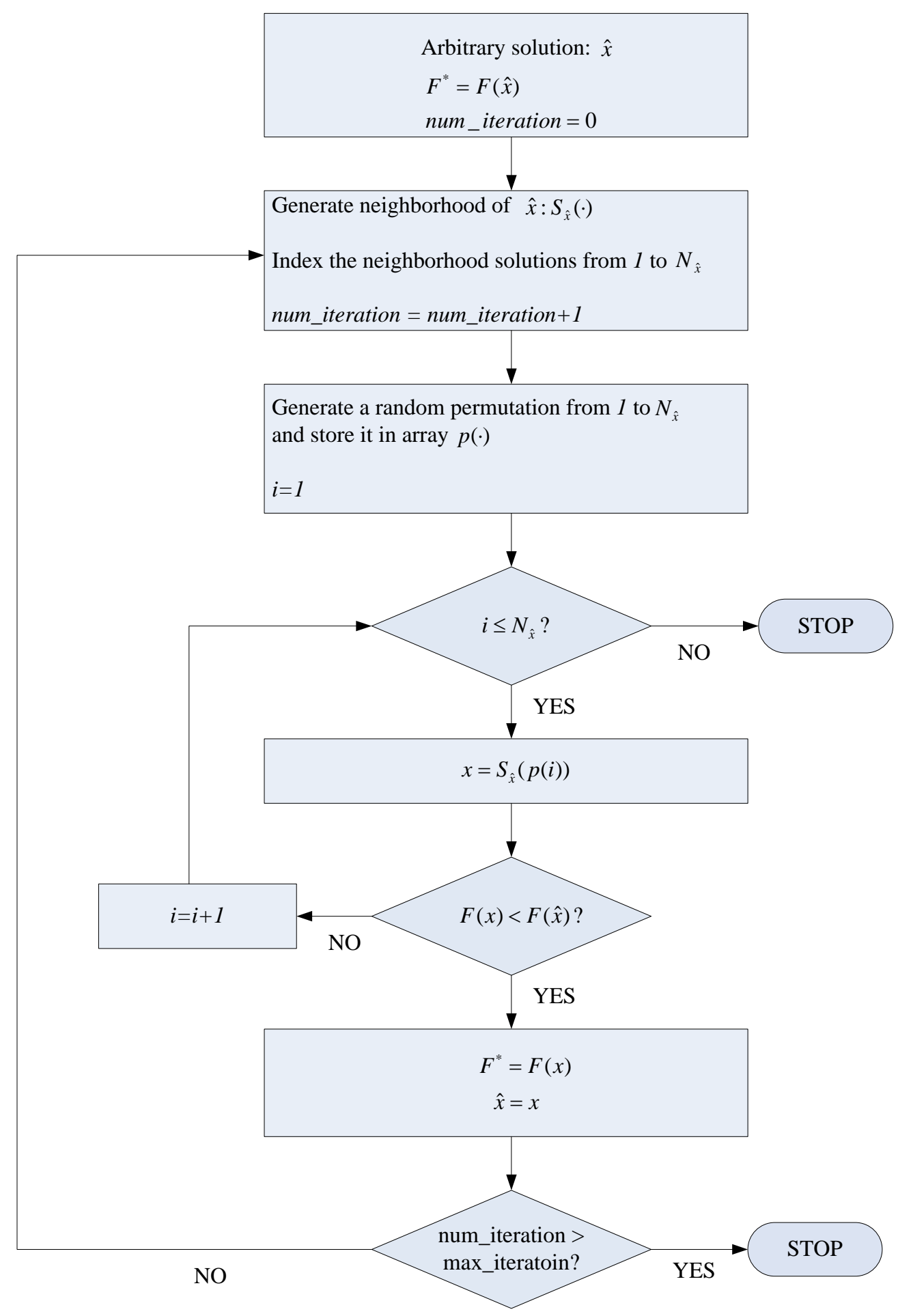

Fig. 5. Flowchart of a simple descent method.

constraint, $D^{\prime} \geq c_{2} x+\left(b-A_{2} x\right)^{t} u_{i}$. At the next iteration, the lower bound must improve or convergence occurs. The following is the explanation. For iteration $i$, we solve (29) with constraints 0 to $i-1$ in (30) and obtain a new integer solution $\widehat{x_{i}}$ and a lower bound value $B_{L}(i)$. In step two, if the upper bound equals the lower 
bound, convergence occurs; otherwise, we proceed to step 3 and generate a new constraint $D^{\prime} \geq c_{2} x+\left(b-A_{2} x\right)^{t} u_{i}$ and a new upper bound $B_{U}(i+1)$. This new constraint, which is a half space, either cut away the solution, $\widehat{x_{i}}$, from the solution space defined by constraints 0 to $i-1$ in (30) or not. If it does cut way $\widehat{x_{i}}$, the optimal objective value of (29) for iteration $i+1$ is going to be better (larger). If it does not, $\widehat{x_{i+1}}$, which is the optimal solution of the next iteration, equals $\widehat{x_{i}}$. Since the upper bound $B_{U}(i+1)$ is smaller than or equal to $c_{2} \widehat{x_{i}}+\left(b-A_{2} \widehat{x_{i}}\right)^{t} u_{i}$ and the lower bound $B_{L}(i+1)$ is larger than or equal to $c_{2} \widehat{x_{i+1}}+\left(b-\widehat{A_{2}} \widehat{x_{i+1}}\right)^{t} u_{i}=$ $c_{2} \widehat{x_{i}}+\left(b-A_{2} \widehat{x_{i}}\right)^{t} u_{i}$, convergence occurs.

The main idea of the Bender's method is to generate constraints iteratively and reduce the solution space gradually until optimality is achieved. The modified Bender's method can be view as an set of additional procedures append to the original Bender's method. Instead of performing a minimization at each iteration, the modified Bender's method uses heuristic to generate a set of solutions and their corresponding constraints rapidly. These set of constraints along with the objective cut constraint (31) may help cut deeper into the solution space (30) and accelerate convergence. If these additional constraints are not helpful, the procedure will perform an minimization and find the useful constraint. Thus, the modified Bender's method will converge to the same value as the original Bender's method.

\section{Wireless Link Capacity Models}

In this section, we discuss practical technologies that enable the heterogenous wireless mesh network, where we will apply the proposed optimization framework. In particular, we give a brief description on IEEE 802.16 technologies. Moreover, we derive the operational bit rate between any two nodes by using the local and back-

bone technologies, i.e., $C_{i j}^{B}$ and $C_{i j}^{L}$, by using information from the IEEE 802.16 PHY specifications and a large scale propagation model.

In practice, there are a few technologies suitable for the backbone and local networks. For backbone networks, long haul highly directional communication is required. Therefore, short wavelength communications systems are very suitable for this network. In this work, we assume that the IEEE 802.16 WirelessMAN-SC technology is used in the backbone network. The WirelessMAN-SC PHY specifications are targeted for operation in the $10-66 \mathrm{GHz}$ millimeter microwave spectrum. Because of such high frequencies, a line-of-sight condition between the transmitter and receiver is required to achieve high quality and available service. Moreover, high performance adaptive antenna array systems are needed to minimize the number of multipaths and interference from unexpected sources. Because of the advance in directional antenna design and the short wavelength used by this technology, interference in the backbone network can be controlled effectively. 
Table 1

\begin{tabular}{|c||c|c|c|}
\hline & QPSK & 16QAM & 64QAM \\
\hline \hline Bit rate $(\mathrm{Mbps})$ & 32 & 64 & 96 \\
\hline Threshold $(\mathrm{dBm})$ & -78 & -71 & -62 \\
\hline
\end{tabular}

Bit rates and signal strength thresholds for a $20 \mathrm{MHz}$ wireless link using IEEE 802.16 WirelessMAN-SC PHY specifications.

Table 2

\begin{tabular}{|c||c|c|c|}
\hline & QPSK-1/2 & QPSK-3/4 & 16QAM-1/2 \\
\hline \hline Bit rate $(\mathrm{Mbps})$ & 16 & 24 & 32 \\
\hline Threshold $(\mathrm{dBm})$ & -86 & -84 & -79 \\
\hline \hline & $16 \mathrm{QAM}-3 / 4$ & $64 \mathrm{QAM}-2 / 3$ & $64 \mathrm{QAM}-3 / 4$ \\
\hline \hline Bit rate $(\mathrm{Mbps})$ & 48 & 64 & 72 \\
\hline Threshold $(\mathrm{dBm})$ & -77 & -72 & -71 \\
\hline
\end{tabular}

Bit rates and signal strength thresholds for a $20 \mathrm{MHz}$ wireless link using IEEE 802.16 WirelessMAN-OFDM PHY specifications.

For local networks, we can use the IEEE 802.16 WirelessMAN-OFDM technology. The IEEE 802.16 WirelessMAN-OFDM PHY specifications are targeted for operation in the 2-11 GHz spectrum. Because it occupies a lower frequency spectrum and because of the multipath fading resilience property of OFDM, IEEE 802.16 WirelessMAN-OFDM is an ideal technology for local networks.

We derive the operational link rates for both IEEE 802.16 technologies in a similar fashion. For both technologies, given the expected received signal strength, the transmitter and receiver will negotiate an appropriate modulation scheme and coding rate. The combination of different modulation schemes and coding rates result in different operational link rates. In a commercial IEEE 802.16 system, which is not widely available in the market yet, additional techniques such as power control and periodic link quality estimation can make the wireless links more reliable. However, these operations are not specified in the standard. The algorithms are proprietary to the venders. Therefore, in this work, we assume a more elementary approach to estimate the operational link rates.

Suppose there are $M$ data rates, denoted $r_{1}, r_{2}, \ldots, r_{M}$, supported by the physical layer. Reliable communication by using rate $r_{m}$ can be realized only if the signal strength at the receiver is above a certain threshold, say $\eta_{m}$. Consequently, for the set of $M$ data rates, there is a set of $M$ thresholds, $\eta_{1}, \ldots, \eta_{M}$. For example, in IEEE 802.16 WirelessMAN-OFDM, there are 6 different bit rates, and the minimal threshold for each bit rate is specified by the standard [24]. The bit rates, signal strength thresholds, and the corresponding modulation/coding schemes for both IEEE 802.16 technologies are summarized in Table 1 and 2. 
We study the case where the following large-scale propagation model [25] is applicable:

$$
P_{r}=\frac{P_{1}}{d^{\alpha}}
$$

where $P_{1}$ is the reference signal power measured at one meter away from the transmitter, $P_{r}$ is the signal power measured at $d$ meters away from the transmitter, and $\alpha$ is a positive constant representing the path-loss exponent. The reference power $P_{1}$ can be obtained via field measurement. Since the exact link adaptation scheme is not specified by the standard, we assume the power control and link estimation schemes are able to maintain the signal strength to a stable value at the receiver. In other words, we assume the operational bit rate of a pair of transmitter receiver can be determined by the receive signal strength calculated by the above large scale propagation model.

\section{Numerical Analysis}

In this section, we present numerical results based on a hypothetical IEEE 802.16 network. The above link capacity model is used to determine the operational bit rate between any pair of nodes. The optimal RS placement and channel assignment in a typical rural environment will be derived by the proposed modified Bender's decomposition method. The runtime of the modified and original Bender's method are discussed.

By using the proposed optimization framework, we evaluate the cost of deploying a heterogeneous wireless mesh network with relay stations in a sparse rural area. We set the infeasibility penalty, $P$ to 1000 . For the backbone network, we use the IEEE 802.16 WirelessMAN-SC technology with transmitter reference power of $10 \mathrm{dBm}$, while for the local network, we use the IEEE 802.16 WirelessMAN-OFDM technology with transmitter reference power of $15 \mathrm{dBm}$. The path-loss exponent for the backbone and local networks are 2.8 and 2.4 respectively. As shown in Fig. 6, the subscribers are distributed in a $12 \mathrm{~km} \times 12 \mathrm{~km}$ rural area. The ISP leases dark fiber from a railroad company and setup its initial base station and central office at node 0 where Internet traffic can be added and dropped into the railroad company's fiber network.

According to the IEEE 802.16 WirelessMAN-OFDM specifications, channel bandwidth can be adjusted dynamically. However, the bandwidth occupied by each channel is vendor specific. In this work, we consider a $20 \mathrm{MHz}$ spectrum is used by the local network, and the occupied spectrum is divided into three channels. ${ }^{6}$ Each RS or base station has a $4 \mathrm{~km}$ interference zone. In other words, if a base station or RS using a particular channel is placed in one location, another RS which

\footnotetext{
6 The link rate of one channel is one third of the original $20 \mathrm{MHz}$ channel.
} 


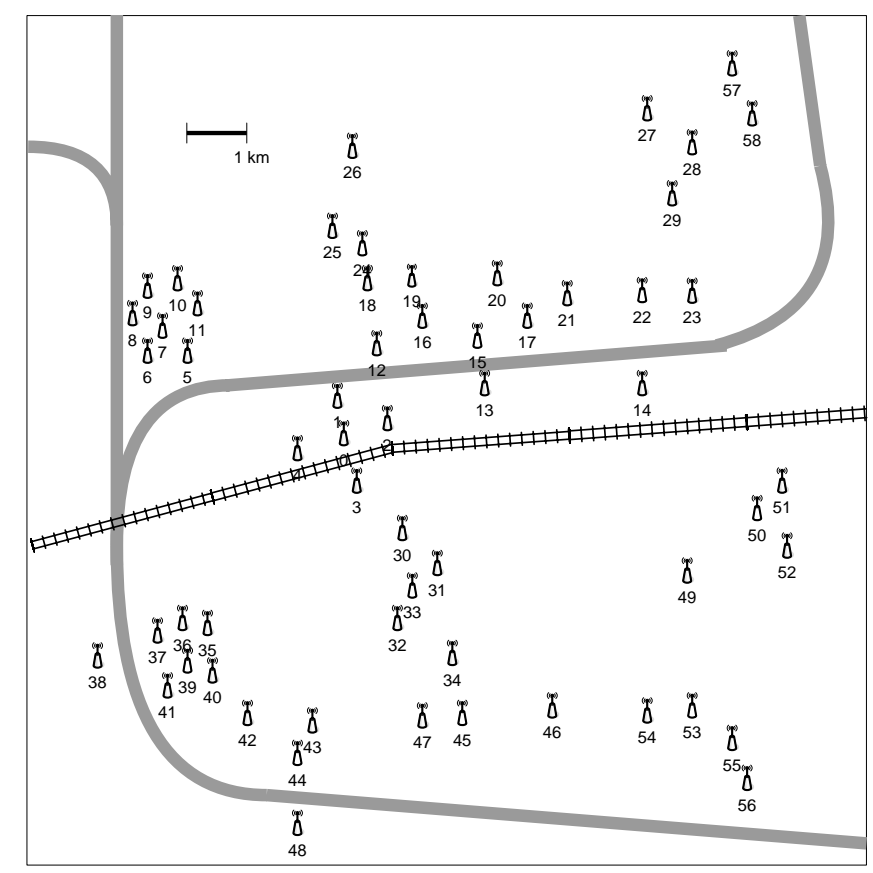

Fig. 6. Network topology used in numerical analysis.

uses the same channel cannot be placed within a $4 \mathrm{~km}$ radius of the former base station or RS. Among the 58 nodes, the ISP has access to 50 of them for the installation of RSs. Furthermore, we set the uplink demand of each subscriber be $1 \mathrm{Mbps}$, while the downlink demand of each subscriber be $2 \mathrm{Mbps}$.

\subsection{Run Time of Original and Modified Bender's Decomposition Method.}

As mentioned in Section 4.3, the original version of Bender's decomposition method requires to solve a small pure $0-1$ optimization problem in each iteration. Even though it is a manageable approach, it can potentially take a long time to perform the computation. In the modified version, we proposed to integrate heuristic and an objective-cut method to reduce the runtime of the Bender's method. For fair comparison, the same Intel Xeon based server with 3.06GHz CPU is used.

The convergence of the original and modified Bender's decomposition methods are shown in Fig. 7, and the configuration of the network is shown in Fig. 8. It takes about 22 hours for the modified version of the Bender's decomposition method to converge to the optimal value, while in the same amount of time, the gap between upper bound and lower bound generated by the original method is still very large. As one can predict from the trend in Fig. 7, it would take at least three to four days for the original bounds to converge. By using the original Bender's decomposition method, the lower bound increases at each iteration since the optimal value of (29) and a desired extreme point are computed for every iteration. While in the modified version of the Bender's decomposition method, a heuristic, which is much faster 


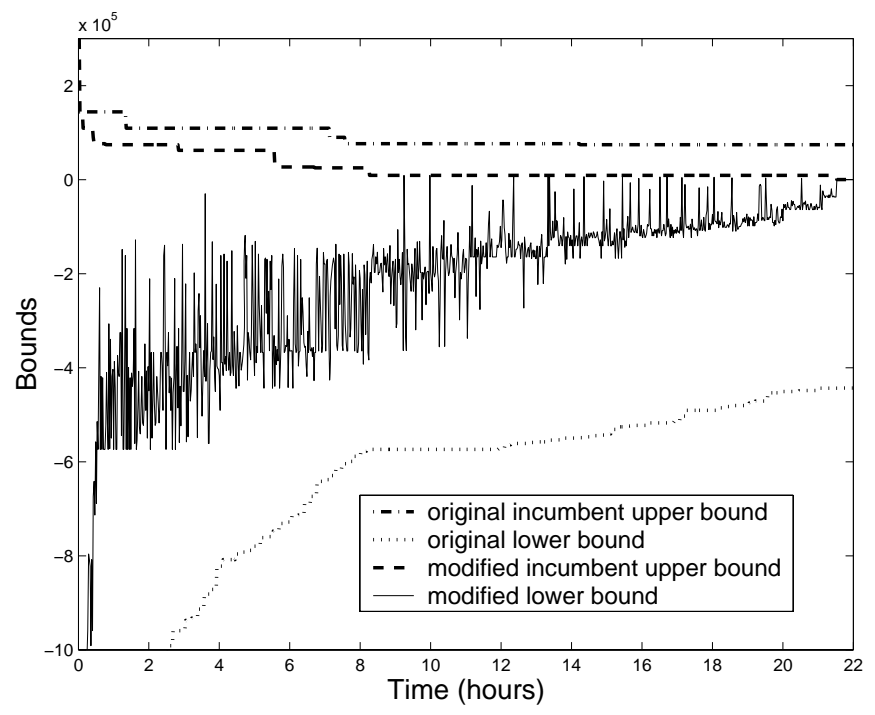

Fig. 7. Convergence of the original and modified Bender's decomposition method.

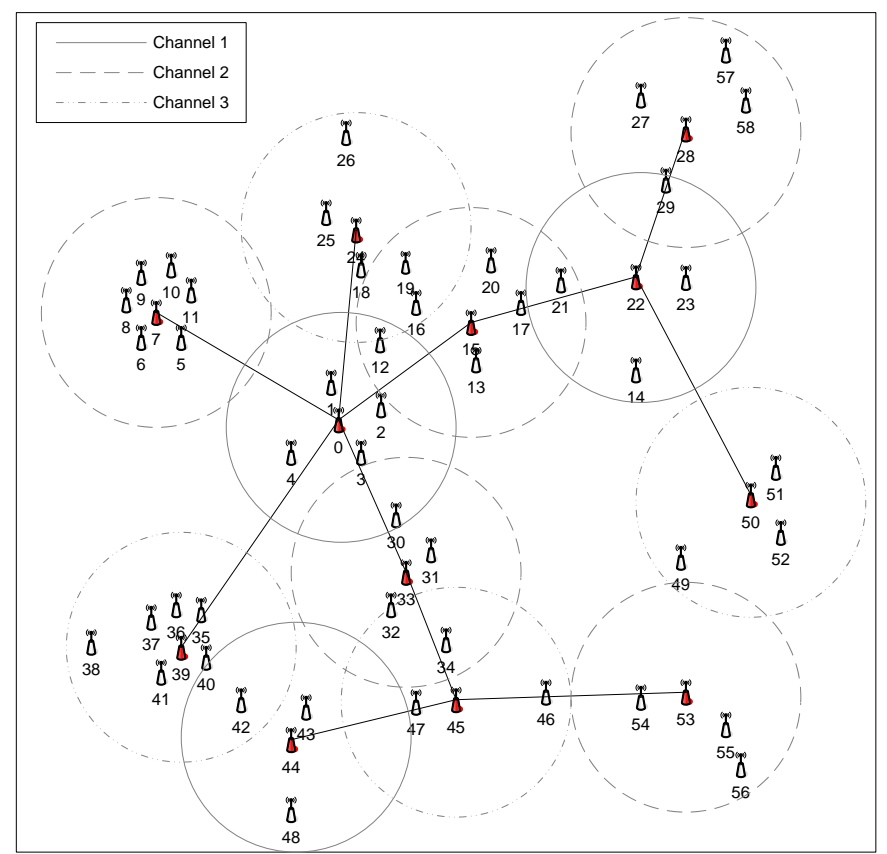

Fig. 8. Network configuration of a heterogeneous wireless mesh network.

than the optimization in the original approach, is use to find a decent solution of (29) and a decent extreme point at each iteration. Since the optimal solution may not be attained at each iteration, the estimated lower bound computed by the modified version fluctuates as shown in Fig. 7. Even though the extreme points generated by the heuristic at some iterations are not the desired extreme points, the heuristic can rapidly generate a set of useful extreme points which leads to faster convergence than the original approach. 


\subsection{Discussion on Pure Heuristic Approaches}

To solve any difficult optimization problem, either a a global optimization algorithm or a heuristic approach can be employed depending on the application. Global optimization techniques are used to solve non-real-time planning problems where long runtime is not a problem, but high quality or optimal solution is required. When the size of the problem is not too large, and the application of the problem is non-real-time, such the RS placement and channel assignment problem described in this chapter, global optimization techniques are preferred. However, heuristics are popular in solving large difficult integer programs. The runtime of heuristics is generally much shorter. However, the quality of a solution generated by heuristics may not be satisfactory in some instances. In addition, there is no way to quantify the quality of a solution produced by heuristics. In other words, the degree of optimality of a calculated solution cannot be obtained. In general, heuristics are used to solve real-time problems which require fast run-time, but an near-optimal solution is adequate.

In this subsection, we discuss the performance of a popular heuristic, simulated annealing [23], which can be used to solve mixed integer problem. Our goal is to shed some insight into the potential of using heuristics to solve the problem of RS placement and channel assignment. For completeness, in the following, we describe the mechanism of simulated annealing within the context of our optimization problem.

To find a solution for the RS placement and channel assignment problem by simulated annealing, we start with an arbitrary RS placement and channel assignment value, $\widehat{x}$, and an acceptance probability, $p$. By using the integer values $\widehat{x}$, we solve the constraint-violation problem $(21)(22)$, and we let $T$ be the sum of the resulting objective value and $|\widehat{x}|_{1} \cdot{ }^{7}$ At this point, we enter an iterative phase. We randomly flip one bit of $\widehat{x}$, to obtain $\widehat{x}^{\prime}$, and solve the constraint-violation problem (21)(22) again using $\widehat{x}^{\prime}$. Similarly, we let $T^{\prime}$ be the sum of the resulting objective value and $\left|\widehat{x}^{\prime}\right|_{1}$. If $T^{\prime}<T$, we set $\widehat{x}=\widehat{x}^{\prime}$ and $T=T^{\prime}$. Otherwise, we set $\widehat{x}=\widehat{x}^{\prime}$ and $T=T^{\prime}$ with probability $p$. After this, we go back to the beginning of the iterative phase and repeat the process. During the process, we keep track of the best solution we obtain so far. Moreover, we fluctuate the acceptance probability $p$ (cooling schedule). We initialize $p$ to a high value, and then we decrease it gradually. When $p=0$, the algorithm becomes a simple descent method. After running the algorithm as a simple descent method for a fix period of time, we set $p$ to the original high value, and decrease it gradually again. The algorithm stops when a pre-specified time limit is reached.

Ten trials of simulated annealing were performed, and each trial was run on the same server for two days. As shown in Table 3, only two out of the ten trials yield

7 The vector norm $|\mathbf{x}|_{p}=\left(\sum_{i} x_{i}^{p}\right)^{\frac{1}{p}}$, for a positive integer $p$. 


\begin{tabular}{|c||c|c|c|c|c|c|c|c|c|c|}
\hline Trial number & 1 & 2 & 3 & 4 & 5 & 6 & 7 & 8 & 9 & 10 \\
\hline \hline Objective Value & 56364 & 31179 & 37185 & 15 & 82011 & 15 & 26016 & 56364 & 32505 & 45012 \\
\hline \hline
\end{tabular}

Table 3

Results obtained by simulated annealing.

a solution without penalties. Moreover, for these two solutions, about $27 \%$ more relay stations are needed when compared with the optimal solution. This means the network configurations generated by this heuristic is $27 \%$ more expansive to build and maintain. This suggests that by computing the optimal solution, which can be generated by the proposed algorithm in a reasonable amount of time, the cost of building and maintaining the network can be significantly reduced.

\section{Conclusion}

In this work, we investigate the optimal placement and channel assignment of wireless relay stations to minimize the operational cost of a wireless mesh network. We have presented a heterogeneous wireless mesh network architecture which uses relay stations to form a backbone and local networks. Furthermore, we have developed an analytical model to investigate whether a particular RS placement and channel assignment can satisfy the user demands and interference constraints. We propose to use Bender's decomposition to compute the optimal number of RSs and their corresponding placement and channel assignment which minimize the operational cost of a heterogeneous wireless mesh network. Furthermore, we present a combination of heuristic and objective-cut method to reduce the runtime of the Bender's decomposition method.

Applying the proposed numerical analysis framework to a sample wireless network based on the IEEE 802.16 standard, we show that by using a few optimally placed RSs, the subscribers' demands can be met. Given a set of network parameters, the proposed framework and optimization technique may offer significant cost advantages, when used by network designers to compute the optimal placement and channel assignment of relay stations, and provide design guidelines on the network setup and maintenance cost estimations.

\section{References}

[1] A. So, B. Liang, Minimum cost configuration of relay and channel infrastructure in heterogeneous wireless mesh networks, in: Proc. of the International IFIP-TC6 Networking Conference, Atlanta, Georgia, 2007.

[2] C. E. Perkins (Ed.), Ad Hoc Networking, Addison-Wesley Longman, 2001. 
[3] Z. Haas, J. Deng, B. Liang, P. Papadimitratos, S. Sajama, Wiley Encyclopedia of Telecommunications, John Wiley \& Sons, 2002, Ch. Wireless Ad Hoc Networks.

[4] Q. Dong, S. Banerjee, B. Liu, Throughput optimization and fair bandwidth allocation in multi-hop wireless LANs, in: Proc. of IEEE INFOCOM, Barcelona, Spain, 2006.

[5] R. Draves, J. Padhye, B. Zill, Routing in multi-radio, multi-hop wireless mesh network, in: Proc. of ACM MOBICOM, Philadelphia, PA, U.S.A., 2004, pp. $114-128$.

[6] R. Draves, J. Padhye, B. Zill, Comparison of routing metrics for multi-hop wireless networks, in: Proc. of ACM SIGCOMM, Portland, OR, U.S.A., 2004.

[7] M. Kodialam, T. Nandagopal, Characterizing achievable rates in multi-hop wireless mesh networks with orthogonal channels, IEEE Transactions on Networking 13 (4) (2005) 868-880.

[8] S. Waharte, R. Boutaba, Tree-based wireless mesh network architecture: Topology analysis, in: Proc. of the First International Workshop on Wireless Mesh Networks (MeshNets), Budapest, Hungary, 2005.

[9] J. Robinson, K. Papagiannaki, C. Diot, X. Guo, L. Krishnamurthy, Experimenting with a multi-radio mesh networking testbed, in: Proc. of first workshop on Wireless Network Measurements (WiNMee), Riva del Garda, Italy, 2005, pp. $271-282$.

[10] K. Jain, J. Padhye, V. N. Padmanabhan, L. Qiu, Impact of interference on multi-hop wireless network performance, in: Proc. of ACM MOBICOM, San Diego, CA, U.S.A., 2003, pp. 66-80.

[11] K. Ramachandran, E. Belding, K. Almeroth, M. Buddhikot, Interferenceaware channel assignment in multi-radio wireless mesh networks, in: Proc. of IEEE INFOCOM, Barcelona, Spain, 2006.

[12] H. Wei, S. Ganguly, R. Izmailov, Z. Haas, Interference-aware IEEE 802.16 WiMax mesh networks, in: Proc. of IEEE VTC, Vol. 5, Dallas, TX, U.S.A., 2005, pp. 3102-3106.

[13] U. Kumar, H. Gupta, S. Das, A topology control approach to using directional antennas in wireless mesh networks, in: Proc. of IEEE ICC, Istanbul, Turkey, 2006.

[14] Y. Chen, C. Chuah, Q. Zhao, Sensor placement for maximizing lifetime per unit cost in wireless sensor networks, in: Proc. of IEEE MILCOM, Atlantic city, NJ, U.S.A., 2005, pp. 1-6.

[15] A. So, B. Liang, Enhancing WLAN capacity by strategic placement of tetherless relay points, IEEE Transactions on Mobile Computing 6 (5) (2007) 522-535.

[16] Y. Bejerano, Efficient integration of multihop wireless and wired networks with QoS constraints, IEEE Transactions on Networking 12 (6) (2004) 10641078.

[17] R. Chandra, L. Qiu, K. Jain, M. Mahdian, Optimizing the placement of internet TAPs in wireless neighborhood networks, in: Proc. of IEEE ICNP, Berlin, 
Germany, 2004, pp. $271-282$.

[18] S. Glapa, Navigating the harsh realities of broadband wireless network economics, Tech. rep., ArrayComm, Inc. (2004).

[19] M. Beaton, Unwiring broadband, technology white paper, Tech. rep., Navini Networks (2002).

[20] D.Sweeney (Ed.), WiMax Operatos's Manual, Building 802.16 Wireless Networks, 2nd Edition, Apress, 2005.

[21] M. Daskin, Network and Discrete Location: Models, Algorithms and Applications, 1st Edition, John Wiley and Sons Canada, Limited, 1995.

[22] L. Snyder, Facility location under uncertainty: A review, IIE Transactions (under revision).

[23] R. K. Martin, Large Scale Linear and Integer Programming, 1st Edition, Kluwer Academic Publishers, 1999.

[24] IEEE standard for information technology, local and metropoliton networks - specific requirements, part 16: Air interface for fixed broadband wireless access systems) specifications, Tech. rep., IEEE Computer Society (2004).

[25] T. S. Rappaport, Wireless Communications: Principles and Practice, 2nd Edition, Prentice Hall, 2001. 